\title{
A catheter friction tester using balance sensor: Combined evaluation of the effects of mechanical properties of tubing materials and surface coatings
}

Røn, Troels; Jacobsen, Kristina Pilgaard; Lee, Seunghwan

Published in:

Journal of the Mechanical Behavior of Biomedical Materials

Link to article, DOI:

10.1016/j.jmbbm.2018.04.023

Publication date:

2018

Document Version

Peer reviewed version

Link back to DTU Orbit

Citation (APA):

Røn, T., Jacobsen, K. P., \& Lee, S. (2018). A catheter friction tester using balance sensor: Combined evaluation of the effects of mechanical properties of tubing materials and surface coatings. Journal of the Mechanical Behavior of Biomedical Materials, 84, 12-21. https://doi.org/10.1016/j.jmbbm.2018.04.023

\section{General rights}

Copyright and moral rights for the publications made accessible in the public portal are retained by the authors and/or other copyright owners and it is a condition of accessing publications that users recognise and abide by the legal requirements associated with these rights.

- Users may download and print one copy of any publication from the public portal for the purpose of private study or research.

- You may not further distribute the material or use it for any profit-making activity or commercial gain

- You may freely distribute the URL identifying the publication in the public portal 


\section{Author's Accepted Manuscript}

A Catheter Friction Tester Using Balance Sensor: Combined Evaluation of the Effects of Mechanical Properties of Tubing Materials and Surface Coatings

Troels Røn, Kristina Pilgaard Jacobsen, Seunghwan Lee

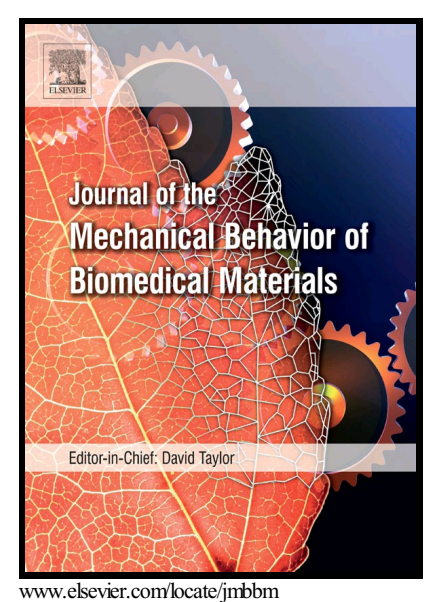

PII: S1751-6161(18)30249-2

DOI: $\quad$ https://doi.org/10.1016/j.jmbbm.2018.04.023

Reference: JMBBM2774

To appear in: Journal of the Mechanical Behavior of Biomedical Materials

Received date: 28 February 2018

Revised date: 6 April 2018

Accepted date: 23 April 2018

Cite this article as: Troels Røn, Kristina Pilgaard Jacobsen and Seunghwan Lee, A Catheter Friction Tester Using Balance Sensor: Combined Evaluation of the Effects of Mechanical Properties of Tubing Materials and Surface Coatings, Journal of the Mechanical Behavior of Biomedical Materials, https://doi.org/10.1016/j.jmbbm.2018.04.023

This is a PDF file of an unedited manuscript that has been accepted for publication. As a service to our customers we are providing this early version of the manuscript. The manuscript will undergo copyediting, typesetting, and review of the resulting galley proof before it is published in its final citable form. Please note that during the production process errors may be discovered which could affect the content, and all legal disclaimers that apply to the journal pertain. 
A Catheter Friction Tester Using Balance Sensor: Combined Evaluation of the Effects of Mechanical Properties of Tubing Materials and Surface Coatings

\author{
Troels Røn ${ }^{1}$, Kristina Pilgaard Jacobsen ${ }^{2}$, Seunghwan Lee ${ }^{1, *}$
}

${ }^{1}$ Department of Mechanical Engineering, Technical University of Denmark (DTU), Kgs. Lyngby DK-2800, Denmark

${ }^{2}$ Department of Electrical Engineering, Technical University of Denmark (DTU), Kgs. Lyngby DK2800, Denmark

*Correspondence: seele@ mek.dtu.dk, +45 45252193

\begin{abstract}
In this study, we introduce a new experimental approach to characterize the forces emerging from simulated catherization. This setup allows for a linear translation of urinary catheters in vertical direction as controlled by a micro-actuator. By employing silicone-based elastomer with a duct of comparable diameter with catheters as urethra model, sliding contacts during the translation of catheters along the duct is generated. A most unique design and operation feature of this setup is that a digital balance was employed as the sensor to detect emerging forces from simulated catherization. Moreover, the possibility to give a variation in environment (ambient air vs. water), clearance, elasticity, and curvature of silicone-based urethra model allows for the detection of forces arising from diverse simulated catherization conditions. Two types of commercially available catheters varying in tubing materials and surface coatings were tested together with their respective uncoated catheter tubing. The first set of testing on the catheter samples showed that this setup can probe the combined effect from flexural strain of bulk tubing materials and slipperiness of surface coatings, both of which are expected to affect the comfort and smooth gliding in clinical catherization. We argue that this new experimental setup can provide unique and valuable information in preclinical friction testing of urinary catheters.
\end{abstract}




\section{Acronyms}

SUM, Silicone-based Urethra Model; CFT-BS, Catheter Friction Tester based on Balance Sensor; PU-Un, Polyurethane-Uncoated; PVC-Un, Poly(vinyl chloride)-Uncoated; PU-C, PolyurethaneCoated; PVC-C, Poly(vinyl chloride)-Coated

Keywords: urinary catheter, friction, flexural strain, urethra model, lubrication

\section{Introduction}

Since urinary tubing is generally made of polymeric materials, they are hardly wet by water, causing high friction, discomfort, and tissue trauma during clinical applications. For this reason, most of catheters are covered with hydrophilic and slippery coatings on the surface in order to assist easy gliding along urethra (Ho et al., 2003; Ikeuchi et al., 1993; Tunney and Gorman, 2002; Uyama et al., 1990; Uyama et al., 1991), although fluidic lubricants such as silicone gels or hydrogels are also widely used (Bardsley, 2005; Cindolo et al., 2004; Gerard et al., 2003; Wilson, 2013). As with other biomedical devices or implants, preclinical testing is essential for urinary catheters too. Since frictional properties are of critical importance for urinary catheters, a number of conventional or specially designed equipment have been employed to characterize the slipperiness of catheters in laboratory (Jones et al, 2001; Røn and Lee, 2016; Stenballe et al, 2005; Tunney and Gorman, 2002). Briefly, there are two major parameters to be considered in the design or selection of friction testing equipment of catheters. The first one is the counter-surface that slides against catheters. In clinical applications, the counter-surface is biological tissues, and more specifically urethra tissue for urinary catheters. Thus, it is most reasonable to provide soft and compliant contacts against catheters, in order to emulate the tribological contacts in clinical applications. A majority of previous studies, however, employed hard materials, such as thermoplastics (Kazmierska et al, 2008) or ceramics (Graiver et al., 1993; Uyama et al., 1990; Uyama et al., 1991), presumably because of convenience and/or availability problem. In this context, we proposed an elastomerbased urethra model with a duct can be a viable approach in a recent study (Røn and Lee, 2016), although further improvement is needed. While animal or human subjects are also employed for pre-clinical testing of frictional properties of catheter (Coveney and Gröver, 2001; Khoury et al., 1991; Nickel et al., 1987; Stenballe et al., 2005), it can be hardly a practical solution due to accessibility issue. The second parameter is the equipment/sensor to probe the emerging forces 
during simulated catherization. Many studies employ texture analyzer setup and probe the forces emerging from sliding contacts between catheter samples and modeled urethra, and they are interpreted as friction forces (Jones et al., 2001; Røn and Lee, 2016; Stenballe et al., 2005; Tunney and Gorman, 2002). Pin-on-disk type tribometer is also a popular approach to probe the resistance of sliding between catheters and modeled friction forces (Graivier et al., 1993; Kazmierska et al., 2008), which is also interpreted as friction forces between them. Both approaches are based on sensing the forces using strain gauge.

In this study, we introduce a new home-built instrumental approach to assess the forces emerging from simulated catherization process in laboratory. The operation principles are similar to those of texture analyzer in the sense that linear movement of catheters in vertical direction is enabled and the resistance from the sliding contacts against model urethra are to be probed. Previously developed silicone-based urethra models with a duct along the cylinder (Røn and Lee, 2016) have been employed in this study too. But, a broader range of parameters, such as environment (ambient air vs water), elasticity of urethra model, clearance and curvature of duct, was varied to investigate the force responses in different experimental conditions. A salient feature of this setup is that a laboratory digital balance is employed as a sensor to probe forces occurring from the sliding contacts between catheter and urethra models (Scheme 1).

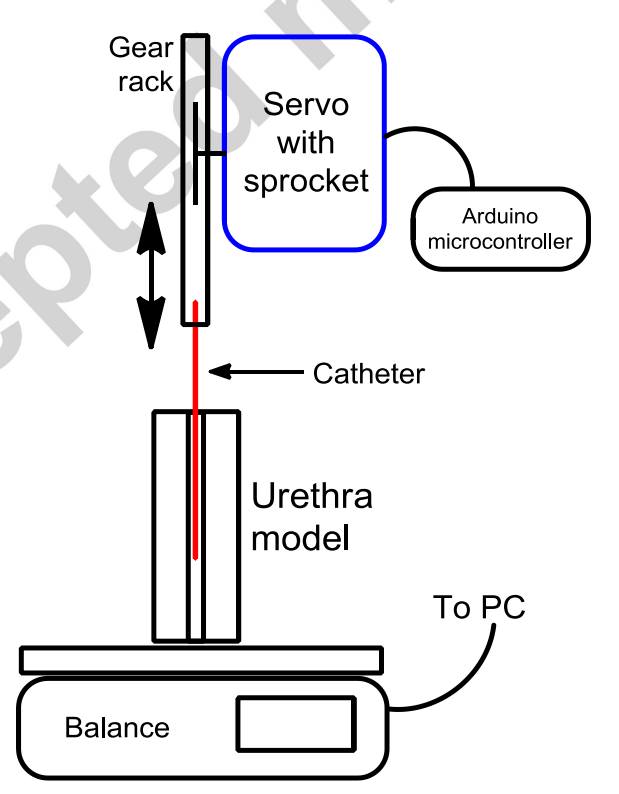

Scheme 1. Schematic illustration of testing setup for measurement of friction forces. 
A photograph of the entire setup is shown in Figure S1 in Supplementary Material. As will be addressed in detail below, this simple, yet unique force sensor allows for the assessment of both mechanical properties of tubing material as well as slippery coatings on the surface.

\section{Materials and Methods}

\subsection{Buffers, chemicals, polymers and catheters}

Agar and polyethylene oxide-block-polypropylene oxide-block-polyethylene oxide (PEO-bPPO- $b$-PEO) triblock copolymer (the type known as "F127" (Wanka et al., 1994)) were purchased from Sigma Aldrich (Brøndby, Denmark) and used without further purification. F127 solution (10\%, weight/vol.) was prepared by dispersing in MilliQ water. PBS tabs purchased from VWR Prolab were dissolved in MilliQ water (resistivity $\geq 18 \mathrm{M} \Omega \cdot \mathrm{cm}$ ) to prepare phosphate buffered saline (PBS) at $\mathrm{pH}$ 7.4. Sylgard 184 and Sylgard 527 polydimethylsiloxane (PDMS) kits were purchased from Dow Corning (Midland, MI). The list of catheter samples in this study is shown in Table 1.

Table 1. List of catheters with specimen code, material, and surface coating characteristics. ${ }^{\mathrm{a}} 4.0 \mathrm{~mm}$ uncoated PU catheters were kindly provided by Coloplast. ${ }^{\mathrm{b}}$ For experiments with $4.7 \mathrm{~mm}$ diameter catheters, the uncoated PU catheters were obtained by rubbing off the PVP coating of "Speedicath ${ }^{\circledR}$ " samples. Lengths of all the catheters were 18 $20 \mathrm{~cm}$.

\begin{tabular}{lllllll}
\hline $\begin{array}{l}\text { Catheter } \\
\text { Code }\end{array}$ & $\begin{array}{l}\text { Commercial } \\
\text { name }\end{array}$ & $\begin{array}{c}\text { Manufacturer } \\
\text { Pulk }\end{array}$ & $\begin{array}{l}\text { Surface } \\
\text { Material } \\
\text { coating }\end{array}$ & $\begin{array}{l}\text { Young's } \\
\text { Modulus } \\
\text { Cervera }\end{array}$ & $\begin{array}{l}\text { Diameter } \\
(\mathbf{m m})\end{array}$ \\
\hline PU-C & SpeediCath $^{\circledR}$ & Coloplast & Polyurethane & PVP & 44.3 & 4.0 \\
PU-Un & SpeediCath $^{\circledR a}$ & Coloplast & Polyurethane & None $^{\mathrm{a}}$ & 44.3 & $4.7^{\mathrm{b}}$ \\
& & & & & 4.0 \\
PVC-C & Curicat $^{\circledR}$ & Curion & PVC & PVP & 17.8 & $4.7^{\mathrm{b}}$ \\
PVC-Un & Pennine $^{\circledR}$ & $\begin{array}{l}\text { Pennine } \\
\text { Pealthcare }\end{array}$ & PVC & None & 17.8 & 4.0 \\
\hline
\end{tabular}


All tested catheters were commercially available except for polyurethane (PU)-based uncoated catheters with $4.0 \mathrm{~mm}$ diameter that were kindly supplied by Coloplast A/S (Denmark). Uncoated PU catheters with $4.7 \mathrm{~mm}$ diameter were obtained from extensive rubbing and cleaning of commercially available PU-based 'SpeediCath ${ }^{\circledR}$, (Coloplast A/S, Denmark) with ethanol to remove coating of hydrophilic poly(vinylpyrolidone) (PVP) layers (SpeediCath ${ }^{\circledR}$ Product Monograph 2010; Madsen, 2010). Hereafter uncoated SpeediCath will be denoted as 'PU-Un' and regular SpeediCath will be denoted as 'PU-C'. Likewise, uncoated PVC catheters from Pennine Healthcare (UK) and PVP-coated PVC catheters (brand name 'Curicat ${ }^{\circledR}$, (Report on Curicat) from Curion (The Netherlands) will be denoted as 'PVC-Un' and 'PVC-C', respectively. The diameters of the catheters were $4.0 \mathrm{~mm}(\mathrm{CH} / \mathrm{FR} 12)$ or $4.7 \mathrm{~mm}(\mathrm{CH} / \mathrm{FR} 14)$ and the length was $18-20 \mathrm{~cm}$. See Table 1 for details.

Solvents were purchased from VWR (Denmark) or Sigma Aldrich (Denmark) and used as received without further purification. Fluorosilane for hydrophobic functionalization of steel rods was trichloro(1H,1H,2H,2H-perfluorooctyl)-silane (Sigma Aldrich).

\subsection{Pin-on-disk tribometry}

The surface friction forces of the catheters were characterized by means of pin-on-disk tribometry. To this end, catheters were circularly mounted on a rotating cup and slid against a flatended PDMS pin under controlled speed and external load. See Figure 1(b) below for more details on the configuration of pin and catheter in this experiment. Flat-ended, cylindrical PDMS pins were prepared with Sylgard 184 kit according to a standard manner described below (see the section 2.3 for details), and BRANDplates ${ }^{\circledR} 96$ microwells C type (VWR) was used as mould. The dimension of flat-ended PDMS pin was $6.1 \mathrm{~mm}$ in diameter and $11.0 \mathrm{~mm}$ in height. Pin-on-disk tribometry experiments were conducted on a commercial pin-on-disk tribometer (CSM Instruments, software version $4.4 \mathrm{M}$, Switzerland). The principal setup of pin-on-disk tribometer consists of a stationary pin pressing down on rotating disk surface. The pin is connected to an arm with a strain gauge enabling measurement of the friction force laterally exerted onto the pin. The load was controlled by applying dead weights on the pin. Friction coefficient $(\mu)$ is calculated according to the formula $\mu=F_{\text {friction }} / W$. The external load $(W)$ was $1 \mathrm{~N}$ in all pin-on-disk experiments, if not stated otherwise. The friction signals for a minimum of 10 rotations were recorded for each measurement to provide $\mu$ average and standard deviation. The cylinder on plane contact model was applied to calculate the mean contact pressure $(P)$ against flat PDMS pin; $P=F / A=F /(2 a L)$, where 
$L$ is the contact length of cylindrical catheter with flat-ended pin $(6 \mathrm{~mm})$, and $a$ is the half width of the line contact and equals as $\left.a=\left(4 \cdot W \cdot\left[\left(1-v^{2}\right) / E_{\text {PDMS }}+\left(1-v^{2}\right) / E_{\text {cath }}\right)\right] \cdot\left(\pi \cdot L \cdot\left[1 / R_{\mathrm{PDMS}}+1 / R_{\mathrm{cath}}\right]\right)^{-1}\right)^{1 / 2}$, where $R_{\text {cath }}$ is radius of catheter $(2 \mathrm{~mm})$ and $R_{\mathrm{PDMS}}$ is infinite for a cylinder-plane contact (Gohar and Rahnejat, 2012; Johnson, 1985). The calculated mean contact pressures in pin-on-disk tribometry under $1 \mathrm{~N}$ were $182 \mathrm{kPa}$ and $189 \mathrm{kPa}$ for PVC and PU catheters, respectively. In this calculation, the reported Young's moduli $\left(E^{\prime}\right)$ of $17.8 \mathrm{MPa}$ and $44.3 \mathrm{MPa}$ for PVC and polyurethane, respectively, by (Cervera et al., 1989) were used, and Poisson's ratios $\left(v_{\mathrm{x}}\right)$ were approximated to 0.5 for PDMS, PVC, and PU. The influence of surface coating and catheters' tubing hole on the reduced Young's modulus $\left(E^{*}\right)$ was ignored.

\subsection{Silicone-based urethra model (SUM)}

PDMS-based urethra models were prepared using commercially available two-component silicone kits, namely Sylgard 184 and Sylgard 527. The base fluid and crosslinker were mixed at 10:1 and 1:1 weight ratios for Sylgard 184 and Sylgard 527, respectively, according to a standard procedure. After mixing, dispersed air bubbles were removed by vacuum, and the fluid mixtures (Sylgard 184, Sylgard 527, or their mixtures) were cast into a $15 \mathrm{~mL}$ polypropylene (PP) centrifuge tube with a vertically positioned stainless steel rod. The sample was cured in an oven $\left(70{ }^{\circ} \mathrm{C}\right)$ overnight. Upon completion of curing and crosslinking of PDMS, the rod was removed and it generated a duct through the center of cylindrical model urethra, where catheters can be inserted for simulated catheterization. The entire process is graphically described in Scheme 2.

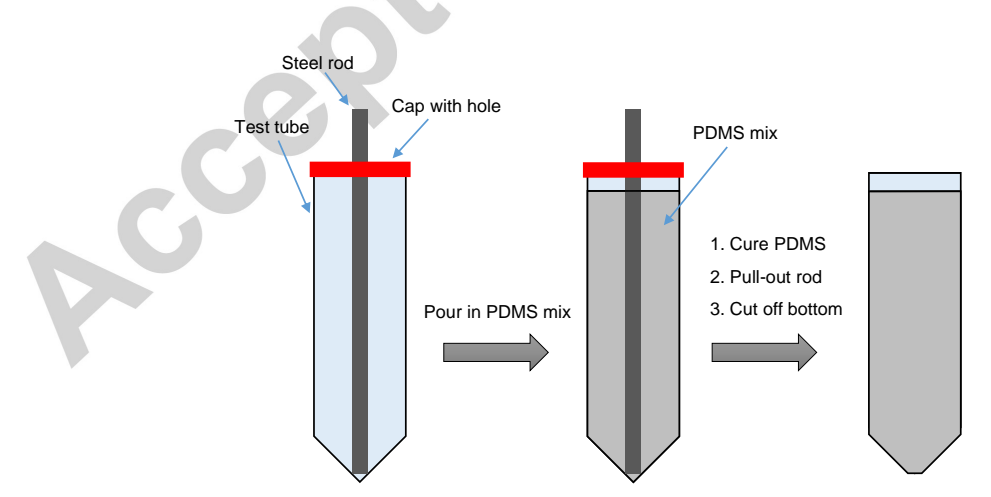

Scheme 2. A schematic illustration of how an in vitro urethra model with castable elastomer (e.g. PDMS) can be prepared. 
Stainless steel rods with different diameters were selected depending on the targeted diameter of duct. Occasionally, the cast PDMS was subjected to $<-20{ }^{\circ} \mathrm{C}$ temperature for easier pull-out of the stainless steel rods. Bent stainless steel rod for casting inflected urethra $(13 \mathrm{~cm}$ radius of curvature, section 3.3.6) was functionalized with fluorosilane for easier release from PDMS in $50 \mathrm{~mL}$ PP centrifuge tube. Briefly, stainless steel rod was cleaned with EtOH (technical gr.) and MilliQ water, then subjected to air plasma treatment at $30 \mathrm{~W}$ for $2 \mathrm{~min}$, and finally was ultra-sonicated in a solution of 10 drops of trichloro $(1 \mathrm{H}, 1 \mathrm{H}, 2 \mathrm{H}, 2 \mathrm{H}$-perfluorooctyl)-silane and 3 drops of chloroform (HPLC gr.) in $50 \mathrm{~mL}$ decalin (technical gr.) solvent. If not stated otherwise, urethra models with a straight, longitudinal duct and a hole at the end were used in the experiments (see Scheme 1). The hole at the end of urethra model was made by cutting the centrifuge tube and PDMS with a knife. The urethra model fabricated in ca. a $15 \mathrm{~mL}$ centrifuge tube was fixed and placed in a larger centrifuge tube (ca. $50 \mathrm{~mL}$ ) in case that the urethra model is exposed to aqueous solutions. Photos of the urethra models are provided in the Supporting Information (Figure S2 in Supplementary Material). Lastly, the surface chemical properties of duct (inner side) can be readily tailored from very hydrophobic to hydrophilic and numerous methodologies to this end are available (Makamba et al. 2003; Zhou et al, 2012). In this study, we have employed F127 Pluronic ${ }^{\circledR}$ solution to hydrophilize the PDMS surface via spontaneous adsorption of F127 copolymer from aqueous solution (Røn et al, 2014).

The Young's moduli of PDMS prepared from Sylgard 184 and Sylgard 527 are reported to be $1720 \mathrm{kPa}$ and $5 \mathrm{kPa}$, respectively, when they are prepared according to the procedure described above (Palchesko, et al. 2012). The Possion's ratio of PDMS is 0.5 (Mark, 2009). The compliance of PDMS can be adjusted by mixing the two PDMS types. To this end, Sylgard 184 and Sylgard 527 at 1:1 and 1:5 weight ratios were further prepared for PDMS urethra models. PDMS prepared from Sylgard 527 alone was mechanically too weak and easily disrupted in the course of pulling out stainless steel rods. We assumed Young's moduli of $830 \mathrm{kPa}$ and $130 \mathrm{kPa}$ for PDMS with 1:1 and 1:5 weight ratios, respectively, as reported by Palchesko et al (Palchesko, et al. 2012). Hereafter we refer to PDMS types as ' $\mathbf{1 8 4}$ ', ' $\mathbf{5 2 7}$ ' or ' $\mathbf{1 : 1}$ ' or ' $\mathbf{1 : 5}$ ' for two pure PDMS and the two mixtures. Models of urethra made from PDMS will be abbreviated as 'SUM', representing 'Silicone Urethra Model' in the following sections.

Agar gel based urethra models were prepared in $50 \mathrm{~mL}$ test tubes by dispersing agar powder in hot water at $10 \mathrm{mg} / \mathrm{mL}$ concentration and letting the gel cool and set for ca. 2 hours. 


\subsection{Catheter Force Tester with Balance Sensor (CFT-BS) and Data Acquisition}

The schematic of the new experimental setup, namely Catheter Force Tester with Balance Sensor (CFT-BS) is illustrated in Scheme 1. Catheters were fixed by immobilizing the drainage port end to a gear rack using scotch tape, whereas the opposite tip was pointing downwards freely. Linear motion of the gear rack was induced by a sprocket on a continuous rotation servo (Parallax 900-00008). The servo was controlled by an Arduino microcontroller. SUMs were placed on an electronic analytical balance (OHAUS model PA2102) with an accuracy of $\pm 10 \mathrm{mg}$ or $\pm 0.0981 \mathrm{mN}$ $(F=m g)$. The catheters were pre-inserted into urethra models' duct by ca. $20 \mathrm{~mm}$ and the balance was set to zero before measurements started. The electronic analytical balance thus could measure both positive (downwards) and negative (upwards) forces after zero/tare. A standard measurement protocol is that, during one test cycle, catheter was approached for 3.5 seconds (46 mm of insertion), paused for 10 seconds, retracted up for 3.5 seconds, and then paused again. The average speed was $13.1 \mathrm{~mm} / \mathrm{s}$. This cycle was repeated four to five times consecutively in order to obtain average and standard deviation for the "peak" and "residual" forces (see Section 3.2 below). The balance was connected to a PC via COM-port using the R232 interface. Software program RealTerm v. 2.0.0.70 was used to record the data of mass transmitted from the balance. Data from balance was measured to transmit at $11.8 \mathrm{~Hz}$, and the data rate was used to establish the time progression. The measurements under each condition were repeated and the most representative results are presented.

\subsection{Contact angle measurements}

Wettability of water on catheter surfaces was characterized with a Ramé-hart goniometer (model 200-F1, Succasunna, NJ) with a software version 2.4.11. A drop (ca. two $\mu$ L) of MilliQ water was applied to the catheter tubing surface and static contact angles were measured after $30 \mathrm{~s}$.

\subsection{Atomic Force Microscopy (AFM) - surface roughness}

A Bruker AFM model Dimension ${ }^{\circledR}$ Edge $^{\mathrm{TM}}$ with NanoScope 8.02 software was employed to characterize the surface morphological features of catheter samples. Surface roughness of the catheters was characterized by acquiring root-mean-square roughness $\left(R_{\mathrm{q}}\right)$ from tapping-mode topographic images over a $100 \mu \mathrm{m} \times 100 \mu \mathrm{m}$ area. Nanoscope Analysis software (v. 1.40) was used to calculate surface roughness. A Bruker NCHV probe (42 N/m, $320 \mathrm{KHz})$ was used. 


\section{ACCEPTED MANUSCRIPT}

\section{Results}

\subsection{Contact Angle Measurements - Hydrophilicity}

Static water contact angles of PU-Un and PVC-Un were $89.1 \pm 5.1^{\circ}$ and $100.9 \pm 1.6^{\circ}$, respectively, and were comparable to the values reported earlier (Balazs, 2004; Kazmierska et al., 2008). The high water contact angles of the uncoated catheters show the inherent hydrophobic surface properties (i.e. low surface tension) of PVC and PU. Static water contact angles on PU-C and PVC-C surfaces in this study were too low $\left(<5^{\circ}\right)$ to be determined exactly, reflecting the highly hydrophilic characteristics of the coatings.

\subsection{Pin-on-Disk Tribometry}

This first tribological experiments of the study concerns the characterization of frictional properties of catheters with conventional pin-on-disk tribometry. This will provide a reference for the results acquired with CFT-BS, as to be presented in Section 3.3. Figure 1 shows the results from the sliding contacts between catheters and PDMS pin in ambient air and in PBS. In pin-on-disk tribometry setup, both friction coefficient $\left(\mu=F_{\text {friction }} / W\right.$, where $W($ Load $\left.)=1 \mathrm{~N}\right)$ and friction force $\left(F_{\text {friction }}=\mu \times W\right)$ can be readily obtained. Both are plotted in Figure 1(a). The linear speed was 15 $\mathrm{mm} / \mathrm{s}$, close to the $13.1 \mathrm{~mm} / \mathrm{s}$ speed in the friction tester in section 3.3. Figure 1(b) shows the schematic of pin-on-disk tribometry highlight the contact between the PDMS pin and catheter.

(a)

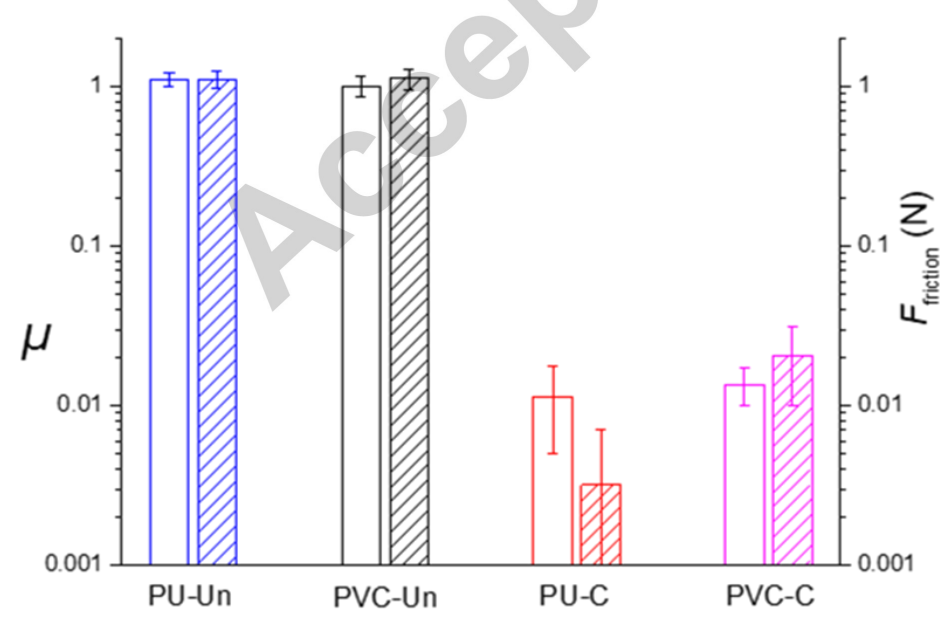

(b)

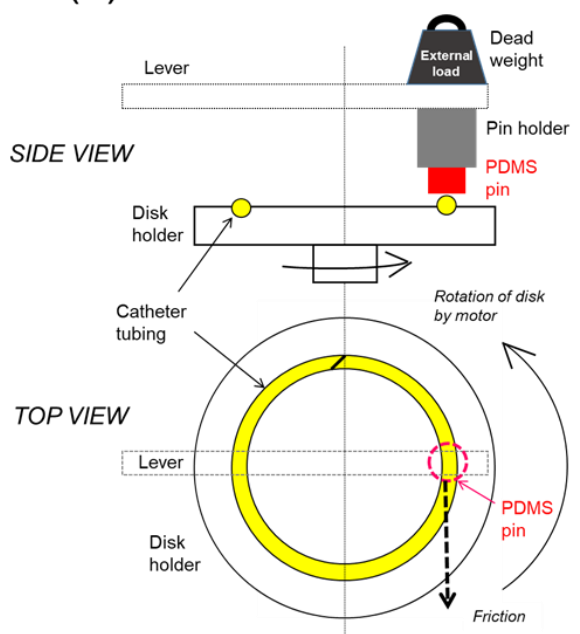

Figure 1. (a) Friction coefficient $(\mu)$ values and friction forces of catheters acquired from sliding against a flat PDMS pin (6.1 $\mathrm{mm}$ in diameter) by means of pin-on-disk tribometry. Error bars are for the standard deviations of $\mu$. Empty 
columns are for dry contacts and shaded columns are for the contacts in PBS (pH 7.4). Linear sliding speed was 15 $\mathrm{mm} / \mathrm{s}$ and external load was $1 \mathrm{~N}$. Minimum 10 laps were measured. (b) Schematic setup of catheter friction testing on pin-on-disk tribometer.

As the catheters were cut to match the circumference of the sliding track, there was a node at the point of junction (see Figure S3 in Supplementary Material) and it caused relatively large friction "negative spikes" in a repeating manner. Thus, the friction outputs from the catheters were filtered by Matlab 'Lowess' function to reduce this effect (see Figure S3 in Supplementary Material). The filtering resulted in ca. 50\% smaller standard deviations, but only ca. 5\% smaller average $\mu$ values. In the following, we demonstrated only the filtered data.

Uncoated catheters' friction coefficients were high in both ambient air and in PBS; for PU-Un, $\mu=1.10 \pm 0.10$ in ambient air and $1.11 \pm 0.14$ in PBS. The $\mu$ values for PVC-Un were $1.00 \pm 0.15$ in ambient air and $1.12 \pm 0.17$ in PBS. Hence, there was no statistical difference in $\mu$ values between the PU-Un and PVC-Un at any conditions. PU-C and PVC-C exhibited much lower $\mu$ values of $\leq 0.02$, i.e. ca. 2 orders of magnitude less than those from uncoated catheters did. Between PU-C and PVC-C, $\mu$ values in ambient air were statistically indistinguishable from each other; $\mu=$ $0.011 \pm 0.006$ and $0.014 \pm 0.004$ for PU-C and PVC-C, respectively. However, in PBS, $\mu$ values of PU-C $(0.003 \pm 0.004)$ were clearly lower than those of PVC-C $(0.021 \pm 0.011)$.

Hydrophobic contacts are known to be adhesive, causing higher effective load $(W)$ and resulting in higher friction (Johnson, 1997; Lee and Spencer, 2005). Pin-on-disk tribometry enables investigation of the adhesive effect by measurement of friction forces with varying load. Figure 2 shows the results of friction force vs. load plots.

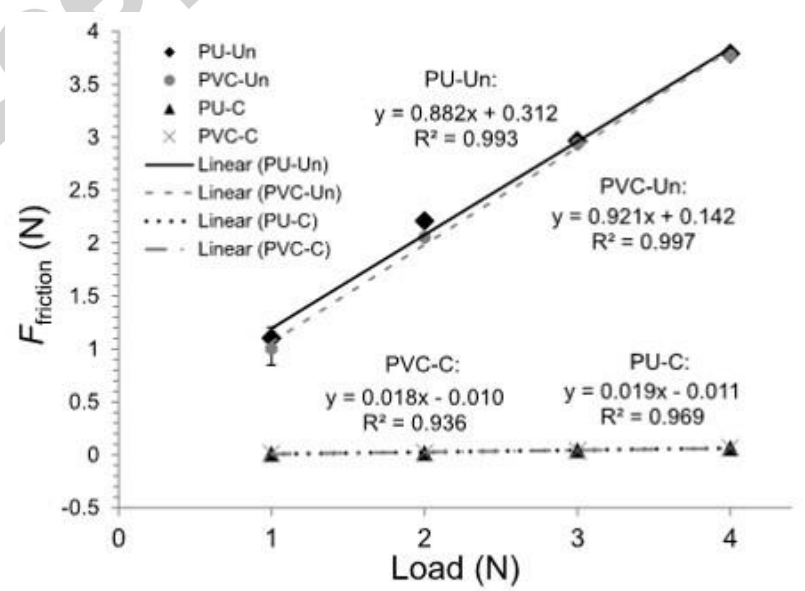

Figure 2. Friction vs. load plots of sliding contacts of a flat-ended PDMS pin on catheter samples as measured with pinon-disk tribometry in ambient air. Average and standard deviation of $F_{\text {friction }}$ were obtained from 10 laps. 
This experiment also shows that the friction forces of PU-Un and PVC-Un are clearly higher than those of PU-C and PVC-C. Additionally, when extrapolating the linear regression to zero load, the friction forces of PU-Un and PVC-Un were non-zero, indicating that the contacts were adhesive in nature. For adhesive contacts, the effective load can be expressed as $W=F_{0}+L$, where $L$ is the applied load and $F_{0}$ is the adhesive normal force at the interface (Deryaguin et al., 1957/58). Hence the friction force is stated as $F_{\text {friction }}=\mu \cdot W=\mu \cdot\left(F_{0}+L\right)$. Consequently, the friction force $\left(F_{\text {friction }}\right)$ under zero applied external load $(L)$ can be entirely attributed to $F_{0}$ and $\mu$. The intercepts of linear regression on the vertical axis in Figure 2 (i.e. at zero load) showed $\mu \cdot F_{0}$ values of $0.312 \mathrm{~N}$ and $0.142 \mathrm{~N}$ for PU-Un and PVC-Un, respectively. PU-Un thus showed somewhat higher adhesive interaction with the PDMS pin than PVC-Un.

\subsection{Catheter Force Tester with Balance Sensor (CFT-BS) against Silicone Urethra Model} (SUM)

\subsubsection{General Observations}

The frictional properties of catheters were characterized by CFT-BS against SUM. Several characteristic features appeared for all types of tested catheters and conditions. Figure 3 displays representative examples of the force output. The vertical axis on the left-hand side represents the measured force (solid lines), and that on the right-hand side represents the relative insertion (dotted lines) where 'zero' insertion is at starting point.

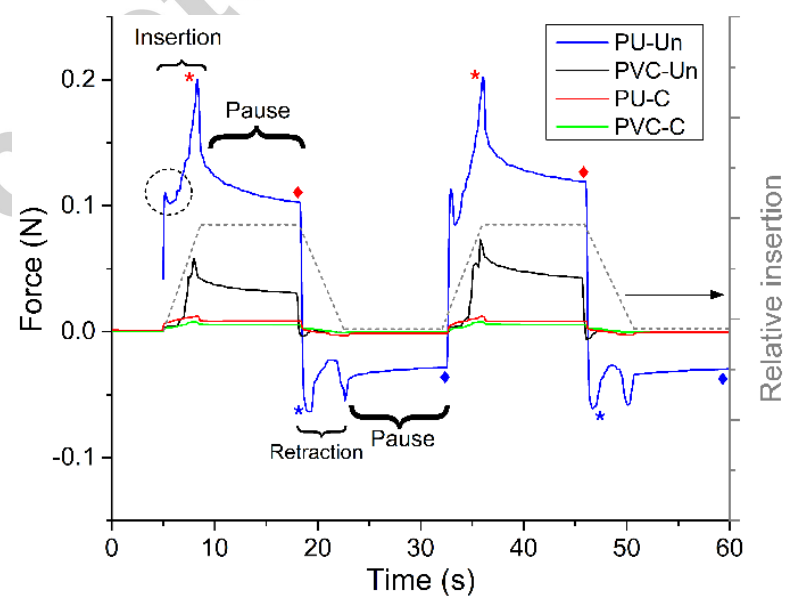

Figure 3. Examples of catheter force measurements of two cycles against a SUM ('184'), in PBS. Right hand side displays the relative insertion of catheter during cycles. Dashed black circle indicates a transition between static and 
kinetic friction from peak to valley. 'Peak forces' are marked with * and 'residual forces' marked with $\bullet$ (red and blue for those during insertion and retraction, respectively).

In the insertion process, the force surged upon initiation of movement, followed by a gradual increase, and finally peaked at the end of the insertion movement. The gradually increasing forces during the entire insertion process cannot be explained by the transition from static to kinetic friction forces. Typically, static friction is larger than kinetic friction, and only the very first dip in the force vs time plot (indicated by the dashed circle) may correspond to this transition. Instead, it can be attributed to gradually increasing area of contact with the progress of insertion (see right hand side axis in Figure 3). This can also explain the fact that the forces are rather gradually decreasing in the retraction process. We designate the maximum force as 'peak force' at the insertion (the difference in forces from zero to $*$ in red). In the subsequent pause for $10 \mathrm{~s}$, the force rapidly decreased first and then reached or converged to a plateau. The observed force plateau at the end of the pause is designated as 'residual force' at the insertion (the difference in forces from zero to $\rightarrow$ in red). The pause intended to mimic the clinical catheterization process where the catheter is stationary e.g. during urination. Residual forces emerge in many cases mainly because the strain applied to catheters during insertion is not released instantaneously at the point of pause. In the case of uncoated catheters, it was often visible that catheters bent slightly during/after insertion. After the first pause, retraction of the catheter up to the starting point commenced, where the force signals converted to negative in sign instantaneously due to the opposite direction of movement. The difference in the residual force at the insertion and the minimum force in the negative part is designated as 'peak force' at the retraction (the difference in forces from $\bullet$ in red to * in blue). At the second pause after the retraction, the catheter returned to the same position at the beginning of the experiment. However, residual forces were non-zero in many cases, for which the absolute values are designated as 'residual force' at the retraction (the difference in forces from $\bullet$ in blue to zero).

In the following sections, averages of peak forces and residual forces over 4-5 cycles, including standard deviations, will be taken as the physical quantities to evaluate force behavior of catheters against various SUMs.

\subsubsection{SUM ('184') with a straight duct: Forces measured in ambient air vs. aqueous environment}

Figure 4(a) shows the CFT-BS results of all four types of catheters (4 $\mathrm{mm}$ in diameter) against the SUM made of '184' with the straight and $5 \mathrm{~mm}$ diameter duct in ambient air. 

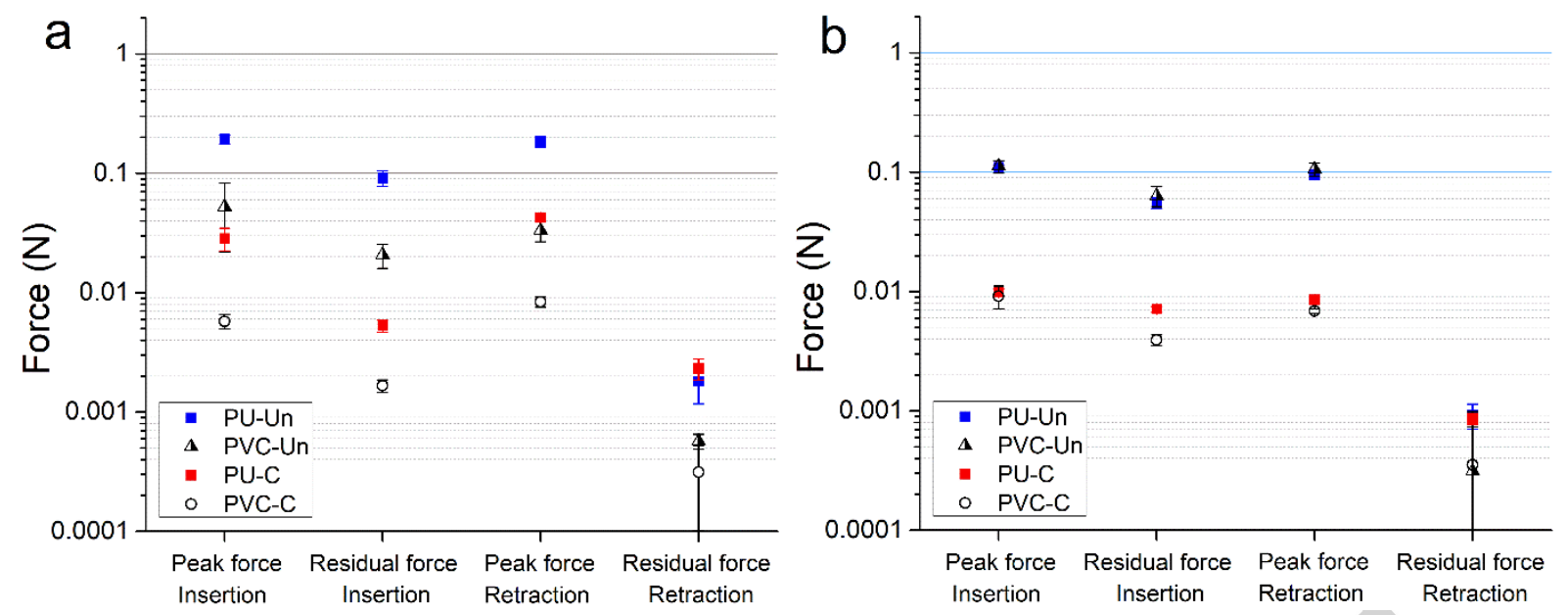

Figure 4. Force measurements of catheters against SUM (184) with a straight duct (5 mm in diameter) and an orifice at the bottom. Data points constitute average of 4-5 measurements with error bars of standard deviation. a) in ambient air. b) in PBS.

PU-Un showed clearly larger forces than PU-C, in terms of peak forces during both insertion $(0.2$ $\mathrm{N}$ vs. $0.03 \mathrm{~N})$ and retraction $(0.2 \mathrm{~N}$ vs. $0.04 \mathrm{~N})$. PVC-Un also displayed larger forces compared to PVC-C (0.05 N vs. $0.006 \mathrm{~N})$. Thus, if we focus on the comparison of uncoated vs. coated cases of individual catheters, the results are very similar to those obtained with pin-on-disk tribometer (Figure 1). However, it should be noticed that PVC-Un and PU-C showed statistically similar peak forces in both directions, and the residual forces in retraction for PVC-Un were rather slightly lower than those for PU-C. Except for residual forces during insertion, the relative order in the forces of sample catheters was PU-Un > PVC-Un $\geq$ PU-C > PVC-C. Thus, clearly distinguished forces between PU-Un and PVC-Un or between PU-C and PVC-C are the features that were observed from CFT-BS experiments only.

In Figure 4(b), forces obtained from the same catheters (4 $\mathrm{mm}$ in diameter) and SUM (184 with the duct of $5 \mathrm{~mm}$ in diameter) as Figure 4(a), yet in aqueous environment (PBS, $\mathrm{pH} \mathrm{7.4)}$ are presented. This set of experiments was performed to mimic the aqueous or moist conditions along the urethra. The forces of PU-C were distinctively smaller for both peak and residual force - during both insertion and retraction - in PBS compared to in ambient air. Meanwhile, the force changes of PVC-C according to environment change were relatively smaller. Thus, even though the average forces of PVC-C were still relatively smaller than those of PVC-Un, the difference between them became much smaller in PBS. Moreover, the peak forces were statistically indistinguishable from each other. We infer that the clearly improved lubricity observed mainly from PU-C in PBS rather 
reflects that the coatings of PVC-C is less sensitive to drying out in ambient air as those of PU-C (at least in the time scale of experiments for Figure 1(a)). Thus, the relative magnitudes of forces between PU-C and PVC-C are clearly different from those observed with pin-on-disk tribometer (Figure 1(a)). For the uncoated catheters, PU-Un and PVC-Un showed identical forces to in all aspects except for residual forces during retraction. Overall, the peak forces were ranked PU-Un $\approx$ PVC-Un $>$ PU-C $\approx$ PVC-C, clearly showing the superior lubricity of the coated catheters than uncoated ones in PBS. Residual retraction forces of all catheters were very low at $\leq 0.001 \mathrm{~N}$.

\subsubsection{PDMS-based SUM vs agar-based urethra model}

Agar gel has often been employed for preclinical catheter friction testing because of its soft characteristics, and thus was employed as a reference model urethra in this study too (Jones et al., 2001; Marmieri et al., 1996; Tunney and Gorman, 2002). Figure 5(a) shows the results from the sliding contacts of catheters against agar gel urethra model as tested with the CFT-BS.

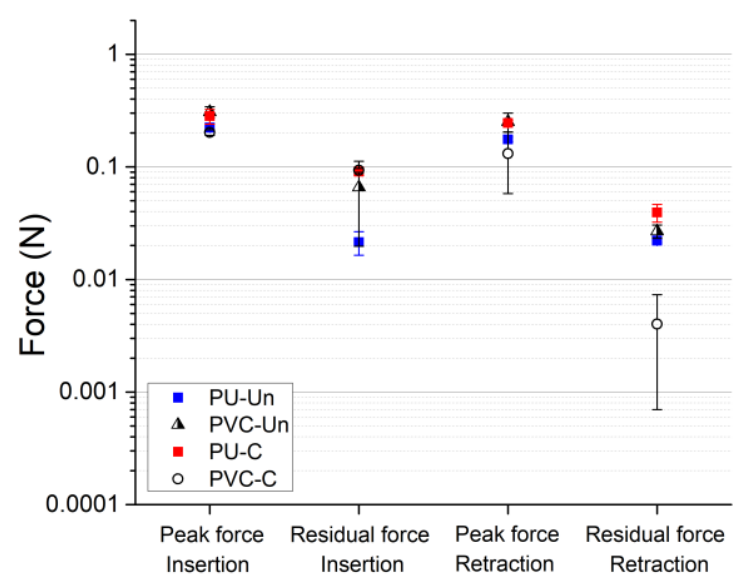

Figure 5. Force measurements of catheters against agar gel with CFT-BS. Data points constitute average of 4-5 measurements with error bars of standard deviation.

Results in Figure 5 are significantly different from those obtained from sliding against SUM, either in ambient air (Figure 5(a)) or in PBS (Figure 5(b)). There was a large degree of overlap in forces in agar model for all catheters at all conditions except for the residual forces of PVC-C during retraction. Furthermore, compared to the movement along a PDMS duct (Figure 5), the difference between residual forces during insertion and retraction were much smaller for most cases. This behavior could be an artifact caused from enveloping or wrapping by agar gel around the catheters, 
affording similar contact areas during both insertion and retraction. Overall, agar gel afforded less sensitive distinction between catheters with varying tubing materials and coatings in general, and poorer distinction of residual forces during insertion vs. retraction in particular.

\subsubsection{Influence of elasticity modulus and duct clearance}

The elasticity modulus of PDMS 184 is still clearly larger than those of most biological tissues, including urethra. Thus, SUMs with lower elasticity modulus, namely '1:1' and '1:5', were employed for further tests in PBS environment. The results are displayed in Figure 6(a) and (b).
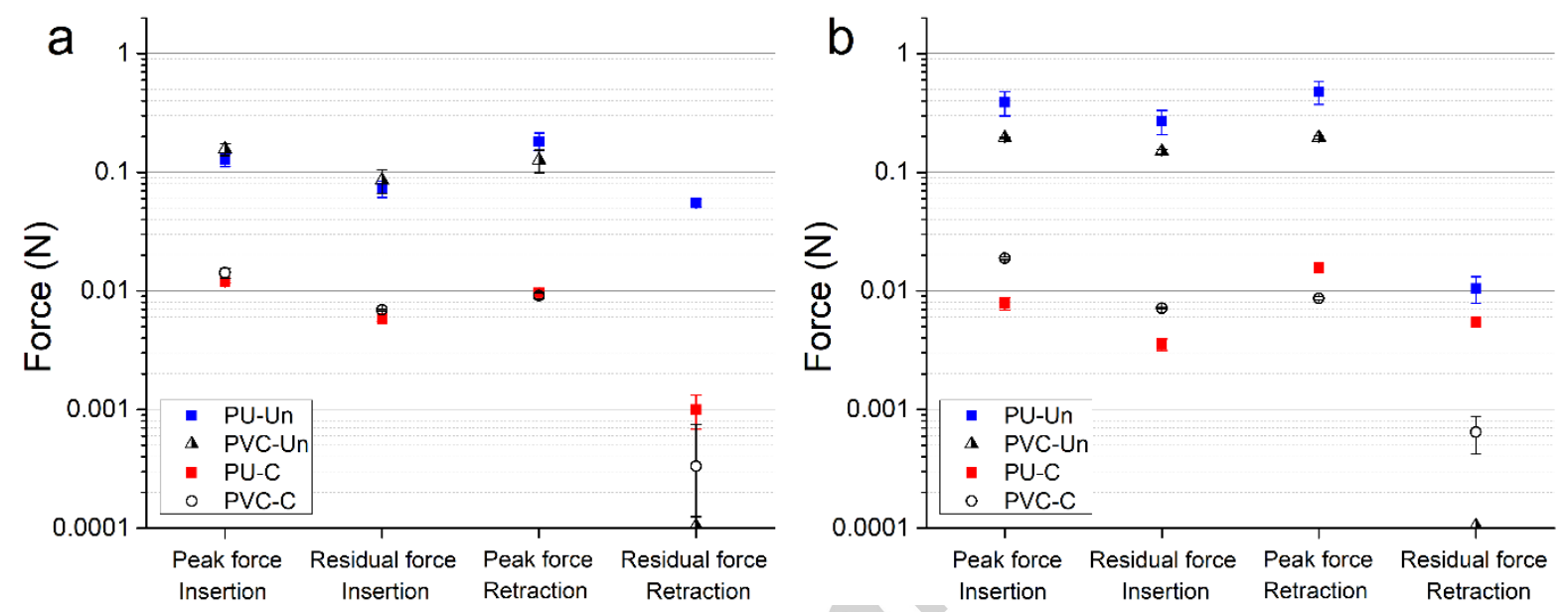

Figure 6. Force measurements of catheters against SUM with a straight duct (5 $\mathrm{mm}$ in diameter) and an orifice at the bottom in PBS in variation of the elasticity modulus of SUM. SUM was a) 1:1 (For PVC-Un the averaged residual force during retraction was lower than the detection limit of $0.0001 \mathrm{~N}$, hence we plot its value is here as the lowest detectable of $0.0001 \mathrm{~N}$ but including the confidence interval of the original standard deviation) b) 1:5 (For PVC-Un the averaged residual force during retraction was lower than the detection limit, hence we plot its value is here as $0.0001 \mathrm{~N}$ ).

Forces against the SUM 1:1 (Figure 6(a)) were similar to those against the SUM 184 in PBS (Figure 4(b)) with an exception of PU-Un, giving significantly larger residual forces during retraction. Forces of catheters sliding against the '1:5' (Figure 6(b)) also showed similar trends with the '184' case, although the forces for both uncoated catheters were somewhat higher. According to Hertzian contract mechanics, a softer counter surface imparts lower effective Young's modulus of the interface and hence affords larger contact area and larger friction forces. This can explain the larger forces of uncoated catheters observed in Figure 6(b). In comparison of PU-Un and PVC-Un, we again observed larger forces associated to PU-Un compared to PVC-Un, similarly as in the 
section 3.3.2 (Figure 4). Meanwhile, for both SUM 1:1 and SUM 1:5 in Figure 6(a-b), the forces of PU-C were almost identical to, and in some cases lower than those of PVC-C.

The diameter of PDMS duct is larger than catheters for all cases in this study. Thus, compressive loading from the SUM on the catheters is not expected. Nevertheless, clearance of catheters along the PDMS duct is as an important parameter that may affect friction forces, because larger contact area and higher friction forces are expected with decreasing clearance. To test this hypothesis, a set of catheters with $4.7 \mathrm{~mm}$ diameter (as opposed to $4.0 \mathrm{~mm}$ diameter in the previous sections) were tested with 1:5 SUM with $5 \mathrm{~mm}$ diameter duct, thus reducing the clearance to only $0.3 \mathrm{~mm}$. Figure 7 shows the results.

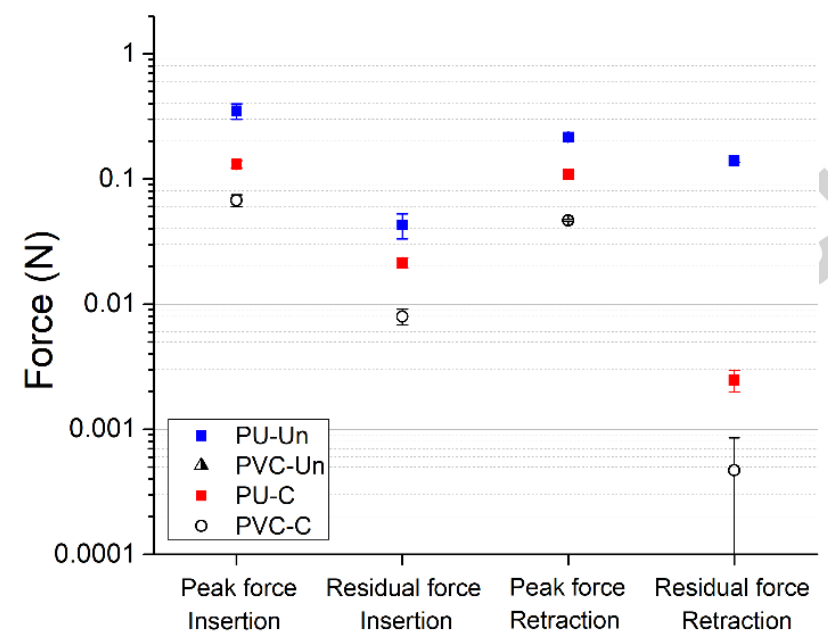

Figure 7. Force measurements of catheters against SUM 1:5 with a straight duct (4.7 $\mathrm{mm}$ in diameter) and an orifice at the bottom in PBS. Resisting force to translate PVC-Un along the duct was too high, thus it was omitted in the plot.

The resistance force for PVC-Un was too high to translate along the duct and the movement of the CTF's gear rack ended up with a bending or buckling of the catheters (thus, data not included in Figure 7). In contrast, PU-Un could go through the duct, despite the larger peak forces than those observed in Figure 6(b). The peak forces of PU-C and PVC-C were also notably larger compared to the case of $1 \mathrm{~mm}$ clearance shown in Figure 6(b). However, the influence of clearance on the residual forces was much smaller for both at insertion and retraction.

\subsubsection{Influence of lubricant and inflection}

So far, SUMs have hydrophobic characteristics along the duct. It is of interest to investigate the force responses when catheters slide along hydrophilic duct, as biological urethra may display hydrophilic characteristics, for instance, when it is wet by urine (Dial et al, 1988). To this end, the 
inner duct of PDMS has to be hydrophilized, but it cannot be readily achieved by conventional approaches, e.g. plasma treatment (Makamba et al. 2003; Zhou et al, 2012) because of geometric restriction. As an alternative, amphiphilic copolymers were employed and dissolved in aqueous solution in which sliding contacts between catheter and SUM take place. Figure 8 displays the experimental results obtained from the catheters with the $4.7 \mathrm{~mm}$ diameter sliding against 1:5 SUM with $0.3 \mathrm{~mm}$ clearance in F127 solution (10 wt\% in water).

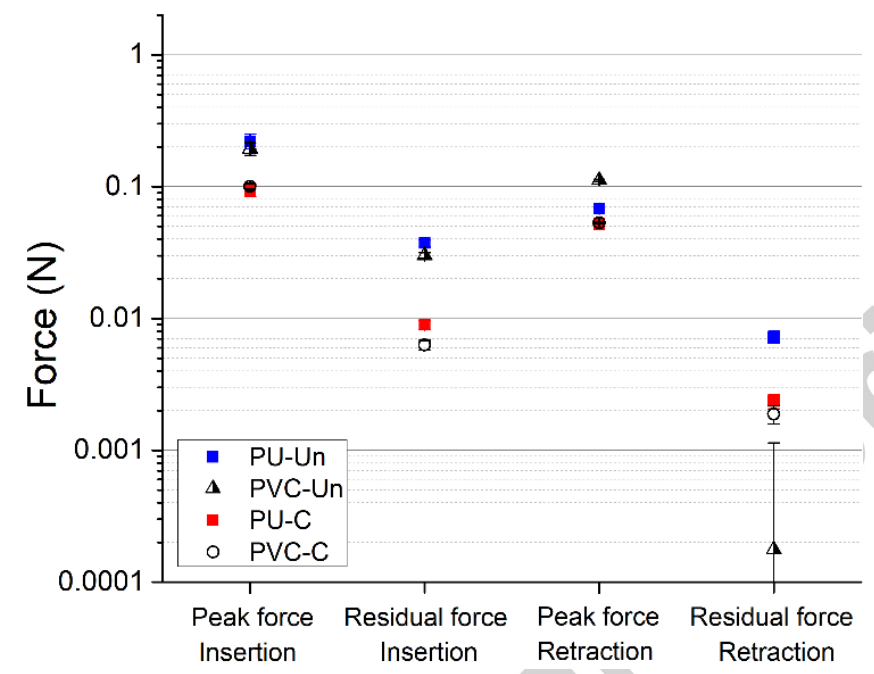

Figure 8. Force measurements of catheters ( $4.7 \mathrm{~mm}$ in diameter) against 1:5 SUM with a straight duct (5 mm in diameter) and an orifice at the bottom in $10 \%$ F127 solution.

As well known, F127 is a triblock copolymer showing amphiphilic characteristics, thus spontaneously adsorbs onto hydrophobic SUM surfaces from aqueous solution and imparts surface hydrophilicity by forming a PEO chain layer (Røn et al, 2014). In aqueous environment, this layer can effectively lubricate the tribological interface by entraining and holding water at the interface. Employing F127 solution lowered the frictional resistance to allow even PVC-Un to enter and move along the duct. For the case of PU-Un, the reduction in forces is fairly evident, presumably due to the coating of PDMS as well as bare PU tubing surfaces. In contrast, it is most notable that the changes in forces for the two coated catheters are much less pronounced. This observation suggests that hydrophilicity of the lubricating films on both catheters may be sufficiently high to wet the gliding interface and reduce the interfacial friction forces.

\subsubsection{Urethra Model - Curved Geometry}


Lastly, we have investigated the force responses of catheters when they move along a nonstraight duct, as inspired from the curved geometry of natural urethra. As a simplest model, a SUM having a duct with well-defined inflection (the radius of curvature $(\mathrm{R})$ was $13 \mathrm{~cm}$ ) was prepared. The PDMS type was 1:5 and the diameter of duct was $5 \mathrm{~mm}$. The catheters with the diameter of 4.7 mm were used in this study. In Figure 9(a), the results obtained from the sliding contacts in PBS are presented.
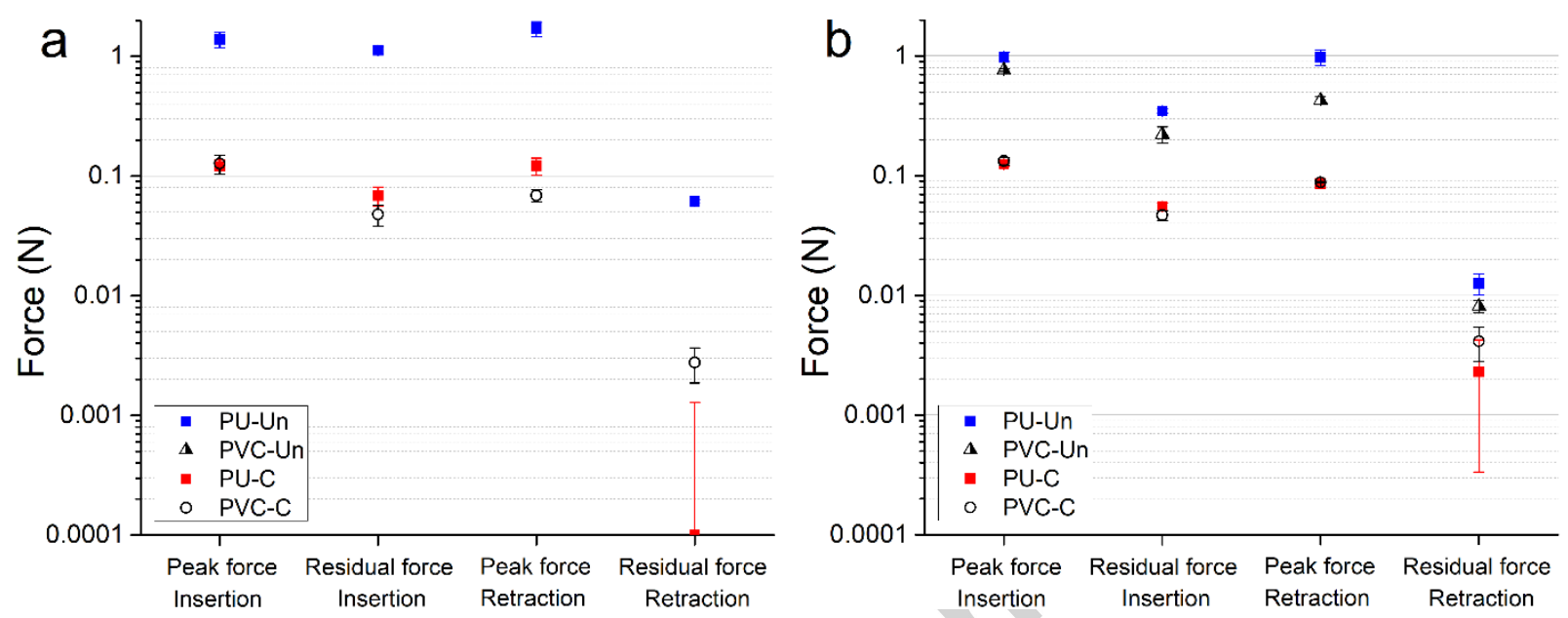

Figure 9. Force measurements of catheters $(4.7 \mathrm{~mm}$ in diameter) against SUM (1:5) with a duct (5 mm in diameter) and a bent inflection ( $\mathrm{R}=13 \mathrm{~cm}$ ) and an orifice at the bottom (a) in PBS (Forces of PVC-Un were too high to move along, and thus omitted in the plot. For PVC-C, the averaged residual forces during retraction were lower than the detection limit of $0.0001 \mathrm{~N}$, hence the value of $0.0001 \mathrm{~N}$ is plotted here, but including the confidence interval of the standard deviation) (b) in $10 \% \mathrm{~F} 127$ solution.

Similarly with the case of straight duct (Figure 7), the resistance force was too high for PVC-Un to move along the duct of SUM. For PU-Un, the peak forces and residual forces during insertion were higher than the case of straight duct (Figure 7) by a factor of ca. $4(1.38 \mathrm{~N}$ vs $0.35 \mathrm{~N})$ and ca. 22 $(1.11 \mathrm{~N}$ vs $0.05 \mathrm{~N})$ on average, respectively. As the duct is bent, while the movement of the catheter is simply linear, larger friction and pushing forces mentioned in the section 3.3 .1 are naturally expected. In contrast, the increase in the peak forces of the two coated catheters during insertion along the bent duct compared to those along the straight duct in Figure 7 was either ignorable for PU-C (118 $\pm 9 \mathrm{mN}$ (straight) vs $111 \pm 16 \mathrm{mN}$ (bent) or only within a factor of 2 for PVC-C (67 \pm 7 $\mathrm{mN}$ vs $127 \pm 23 \mathrm{mN}$ ). Peak forces at retraction showed a similar trend. This implies an effective lubricating performance of the coatings in the initial insertion step even in the bent duct in this study. However, the corresponding residual forces along the bent duct were much larger than those along the straight duct; by a factor of 3.2 for the case of PU-C $(21.4 \pm 2.0$ vs $68.6 \pm 12.1 \mathrm{mN})$ and 


\section{ACCEPTED MANUSCRIPT}

by a factor of 6.0 for the case of PVC-C $(8.0 \pm 1.1 \mathrm{vs} 47.7 \pm 9.4 \mathrm{mN})$. Thus the observed increase in the residual forces is relatively greater than that in peak forces, probably due to larger extent of pushing in the bent case.

Experiments in $10 \%$ F127 solution were carried out in order to characterize the forces along the SUMs, similarly with the section 3.3.5, yet with a bent duct. The results are shown in Figure 9(b). Measurements with PVC-Un were again possible and both peak and residual forces of PU-Un were smaller than those in PBS (Figure 9(a)), which further demonstrates the lubricating effect of F127 on hydrophobic surfaces. Furthermore, PVC-Un displayed smaller forces in all peaks and residual forces compared to PU-Un. Meanwhile, PU-C and PVC-C displayed virtually the same forces as those in PBS (Figure 9(a)) only with slightly larger residual forces at insertion. We infer that this is due to that surface coatings on the coated catheters already provide sufficient lubricity in peak forces. The stiffer catheter counteracts the bending when inserted into a duct with curved geometry as the flexural force is proportional to the Young's modulus of the bent entity, causing more pushing force translated to the balance (Young et al., 2012).

\section{Discussion}

An outstanding advantage of the CFT-BS setup compared to conventional pin-on-disk tribometry in this study is its substantially superior capabilities to distinguish different types of samples catheters, i.e. according to both surface coatings and tubing materials. This is mainly because while pin-on-disk tribometry probes the interfacial friction forces only, the CFT-BS probes 'pushing force' as well by employing a digital balance as force sensor. A diagram of the forces emerging from the contacts between catheters and the inner wall of the duct in SUM is shown in Scheme 3. 
IDEAL SYSTEM WITH NO FRICTION ONLY STATIC PRESSING/PUSHING

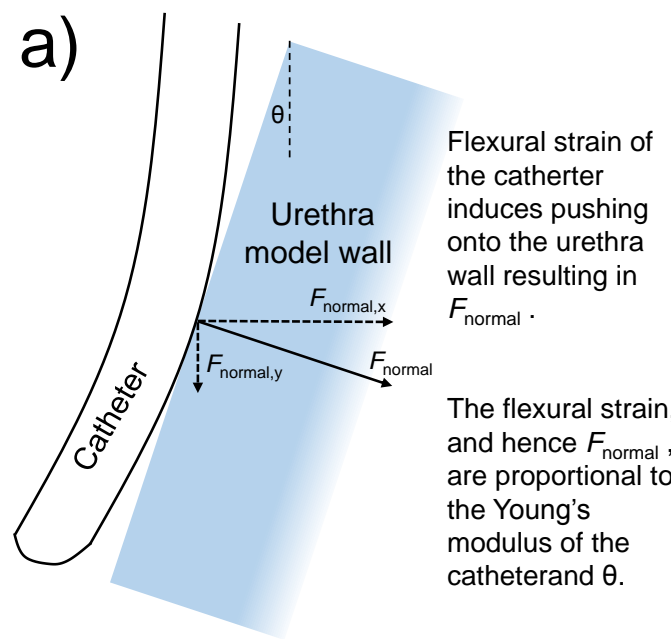

Total force down in y direction:

$$
F_{\mathrm{y}}=F_{\text {normal,y }}=F_{\text {normal }} \cdot \sin (\theta)
$$

REAL SYSTEM WITH FRICTION \& STATIC PRESSING/PUSHING

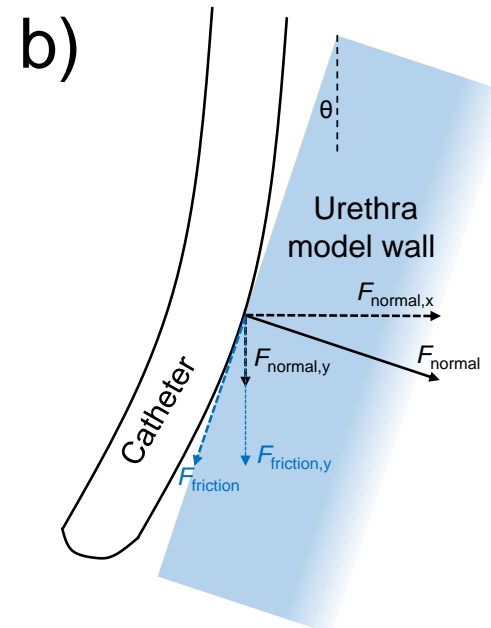

Friction force is always parallel to the surface

Friction part will be larger than 'pressing' part due to scaling. $F_{\text {friction,y }}$ is proportional to $\cos (\theta)$. $F_{\text {normal,y }}$ is proportional to $\sin (\theta) . \theta<10^{\circ}$

Total force down in y direction:

$$
\begin{aligned}
F_{\mathrm{y}}=F_{\text {normal }, y}+F_{\text {friction }, y} & =F_{\text {normal }} \cdot \sin (\theta)+F_{\text {friction }} \cdot \cos (\theta) \\
& =F_{\text {normal }} \cdot \sin (\theta)+F_{\text {normal }} \cdot \mu \cdot \cos (\theta)
\end{aligned}
$$

Scheme 3. Force diagram of catheter in contact with silicone-based urethra model wall. $\theta$ angle is exaggerated for illustrative purpose. (a) Force diagram for an ideal system without friction. (b) Force diagram for real system with friction. Balance only measures forces in y direction.

We define 'pushing force' as the vertical component of the forces acting along the SUM duct's side walls (thus, effectively normal to the balance, see Scheme 3) in the absence of friction. In other words, pushing force acts as additional mass applied on the balance simply from pushing the catheters in normal direction to the balance (lateral to SUM duct wall), yet is not caused from rubbing against the SUM. The pushing force emerges from flexural strain and imperfect parallel alignment of catheters and/or wiggling of catheters along the SUM duct during translation. During translation, while only the normal component is probed by the balance for both pushing force and friction force, the friction part is the dominant force in most cases, as $F_{\text {friction,y }}$ is proportional to $\cos (\theta)$ whereas $F_{\text {normal,y }}$ is proportional to $\sin (\theta) . \theta$ is exaggerated in Scheme 3 for clarity, but it is $\theta$ $<10^{\circ}$ in reality. Pushing forces are thus more apparent in the experiments with a bent duct in SUM (Section 3.3.5) where $\theta$ becomes much larger.

The CFT-BS's capabilities to probe forces including pushing force are ascribed to its unique design that employs a balance as force sensor. As shown Figure 1, conventional pin-on-disk tribometry could not detect any meaningful difference between the two types of catheters, especially between the two uncoated catheters. In fact, interfacial frictional properties of 


\section{ACCEPTED MANUSCRIPT}

engineering polymers are hardly discernable from each other in general, except for very unique examples such as Teflon, because of their commonly hydrophobic surface characteristics (Ikada and Uyama, 1993). Nevertheless, higher forces detected from PU-Un than PVC-Un as observed by CFT-BS suggest that there is other factor that is active under CFT-BS setup only and affects the overall forces. We propose that it is most likely to be bending strain or flexural strain of the catheters when translated along the PDMS duct wall. One extreme example of pushing force suggested above is the mass (force) detected when catheter presses down the balance without even involving SUM. In order to test this conjecture, bending strain of catheters were characterized while directly pressing down on the balance (Figure 10).

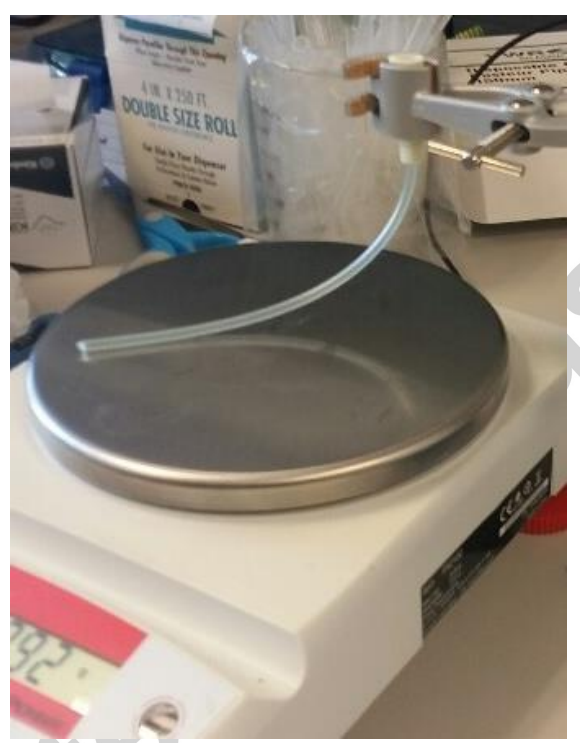

Figure 10. A photograph showing the measurements of bend (flexural) strains of catheters.

In these experiments, the initial height of catheters was adjusted so that a bend by $90^{\circ}$ can be reached at the end of translation. The mass (force) was recorded 13.5 seconds after the initiation of movement, which is identical with the time duration for CFT-BS experiments. Two different PUUn and PVC-Un catheters were employed and five measurements were carried out per each catheter for statistical analysis. The forces were observed to be high in the beginning and then decreased subsequently as in the 'plateau' force section (raw data not shown). The results show that a $90^{\circ}$ bending force of PU-Un was $101 \pm 8 \mathrm{mN}$, and that of PVC-Un was $42 \pm 10 \mathrm{mN}$, i.e. the flexural strain force of PU-Un was statistically twice as high as that of PVC-Un. Ultimately, this difference can account for the generally higher forces observed from the uncoated catheters made of PU than PVC throughout the study. The flexural strain force of catheters was closely linked to 
Young's moduli of tubing materials; 44.3 MPa for PU and 17.8 MPa for PVC (Table 1). As flexural strain force of a tubing is proportional to its Young's modulus, the larger normal force acting on the PDMS duct wall can be ascribed to the higher elasticity modulus of PU compared to PVC as well.

The reason why pushing force is activated even for straight duct (Figure 4 - Figure 8) is that the catheters are never perfectly straight by themselves, and thus the net effect is similar with when a perfectly straight catheter passes through a curved duct. In most other friction test methods of urinary catheters, the role of mechanical properties of tubing materials tended to be ignored and the focus was placed mostly on the assessment of slipperiness of coatings on the surface. This approach is valid and useful to evaluate the lubricating properties of slippery coatings on the catheter surfaces only. However, pain or discomfort for the patients in clinical catherization process arises not only from interfacial friction but also from nominally applied force on the tissues by catheters, especially as urethra is not a simple, straight duct. In this sense, we argue that CFT-BS is an experimental approach that allows for the assessment of urinary catheters in a closer condition to clinical catherization than other experimental approaches. The role of pushing force is important also for coated catheters because the forces detected by CFT-BS from the coated catheters are a combination of friction force and pushing force. In this study, all the CFT-BS experiments were carried out in PBS in variation of other parameters, except for Figure 4(a). As the $\mu$ values of PU-C are clearly lower than PVC-C in PBS as assessed by pin-on-disk tribometry (Figure 1), similar trend should have been observed if interfacial friction forces were dominant in the measurements with CFT-BS too. Thus, comparable forces of PU-C and PVC-C (Figure 4(b), Figure 6(a), Figure 8, Figure 9(a), Figure 9(b)) or even higher forces of PU-C than PVC-C (Figure 6(b), Figure 7) as characterized with CFT-BS can be ascribed to higher flexural strain of PU. Of course, clearly higher forces from PU-C than PVC-C as probed by CFT-BS in ambient air (Figure 4(a)) in contrast to the comparable friction forces between them as probed by pin-on-disk tribometry (Figure 1(a)) can also be accounted for the activation of pushing force in the former experimental setup. Overall, even for catheters coated with hydrophilic and slippery coatings, the forces probed by CFT-BS are a reflection of combined effects of the mechanical properties of tubing materials and the lubricating properties of surface coatings. We argue that this is a very unique feature of the new test method developed in this study and it can provide valuable information in pre-clinical assessment of urinary catheters.

\section{Conclusions}


In this study, we have developed a new friction testing rig (termed as 'catheter force tester using balance sensor' (CFT-BS)) for urinary catheters based on reciprocal movement of catheters along a duct made of silicone-based urethra model (SUM). A most unique feature of the instrumental setup was that a regular digital balance was employed as force sensor by placing it underneath a catheter/SUM assembly. Thus, both friction forces arising from rubbing as well as simple mechanical pressing of catheters against inner duct of SUM, termed as pushing forces, during translation of catheters along the duct of SUM are collectively detected in this setup. Capabilities to detect both forces is meaningful and important because discomfort or tissue trauma during clinical catheterization can be caused by either or the combination of them. Two types of catheters with varying tubing materials (PU vs. PVC) and hydrophilic polymer coatings, along with their respective uncoated catheters, were tested in this study. The CFT-BS provided a high time resolution in force measurements, permitting the distinction between peak and residual forces during insertion and pause, respectively. Moreover, it was possible to give a variation in a number of parameters for SUM, including environment (ambient air vs. PBS), elastic modulus, clearance, hydrophilicity of inner duct, and curvature of duct. In general, force measurements against SUM provided better distinction of catheters with different tubing materials and surface coatings than agar gel model. As expected, catheters with hydrophilic polymer coatings showed lower forces than uncoated catheters. While this feature was easily verified with a conventional pin-on-disk tribometry too, CFT-BS could detect additionally the contribution of the mechanical properties of tubing materials in overall forces. This was possible because pushing forces are originated from the flexural strain of catheters, which are related to the elasticity moduli as well. Higher forces observed from translation of PU-based catheters along the duct of SUM, with or without hydrophilic coatings, than PVC-based catheters were well correlated with the larger flexural strain forces of PU than PVC. We argue that collective measurements of friction forces and pushing forces, which were enabled in CFT-BS setup, can offer pre-clinical evaluation of catheters under much closer conditions with clinical catherization than other conventional experimental approaches.

\section{Acknowledgements}

This study was financially supported from Danish Council for Independent Research (Funding scheme: Individual Postdoc Grant, Project Number 5054-00161) and European Research Council (Funding scheme, ERC Starting Grant 2010, and the project number 261152). 


\section{ACCEPTED MANUSCRIPT}

\section{References}

Bardsley, A., 2003. Use of lubricant gels in urinary catherization. Nursing Standard 20, 41-46.

Balazs D.J., Triandafillu, K., Chevolot, Y., Aronsson, B.O., Harms, H., Descouts, P., Mathieu, H.J., 2003. Surface modification of PVC endotracheal tubes by oxygen glow discharge to reduce bacterial adhesion. Surf. Interf. Anal. 35, 301-309.

Cervera, M., Dolz, M., Herraez, J.V., Belda, R., 1989. Evaluation of the elastic behaviour of central venous PVC, polyurethane and silicone catheters. Phys. Med. Biol. 34, 177-183.

Cindolo, L., Palmieri, E.A., Autorino, R., Salzano, L., Altieri, V., 2004. Standard versus hydrophilic catheterization in the adjuvant treatment of patients with superficial bladder cancer, Urol. Int. 73, 19-22.

Coveney, V.A., Gröver, D., 2001. An abdominal wall simulator for testing suprapubic urinary catheters, Physiol. Meas. 22, 505-516.

Deryaguin, B.V., Karassev, V.V., Zakhavaeva, N.N., Lazarev, V.P., 1957/58. The mechanism of boundary lubrication and the properties of the lubricating film. Short- and long-range action in the theory of boundary lubrication. Wear 1, 277-290.

Dial, E.J., Huang, J., O'Neil, R.G., Hills, B.A., Lichtenberger, L.M., 1988. Surface hydrophobicity and water transport of the toad urinary bladder: effects of vasopressin. J. Membr. Biol. 106, 119122.

Gerard, L.L., Cooper, C.S., Duethman, K.S., Gordley, B.M., Kleiber, C.M., 2003. J. Urol. 170, $564-567$.

Gohar R., Rahnejat, H., 2008. Fundamentals of Tribology, Imperial College Press, London. 


\section{ACCEPTED MANUSCRIPT}

Graivier, D., Durall, R.L., Okada, T., 1993. Surface morphology and friction coefficient of various types of Foley catheter. Biomaterials 14, 465-469.

Ho, S.P., Nakabyayashi, N., Iwasaki, Y., Boland, T. , LaBerge, M., 2003. Frictional properties of poly(MPC-co-BMA) phospholipid polymer for catheter applications. Biomaterials 24, 5121-5129.

Ikeuchi, K., Takii, T., Norikane, H., Tomita, N., Ohsumi, T., Uyama, Y., Ikada, Y., 1993.Wear 161, $179-185$.

Johnson, K.L., 1985. Contact Mechanics, Cambridge University Press, Cambridge.

Johnson, K. L. 1997, Adhesion and friction between a smooth elastic spherical asperity and a plane surface. Proc. R. Soc. Lond. A 453, 163-179.

Jones, D.A., Carvin, C.R., Gorman, S.P., 2001. Design of a simulated urethra model for the quantitative assessment of urinary catheter lubricity. J. Mater. Sci.: Mater. Med. 12, 15-21.

Kazmierska, K., Szwarst, M., Ciach, T., 2008. Determination of urethral catheter surface lubricity. J. Mater. Sci.: Mater. Med. 19, 2301-2306.

Khoury, A.E., Olson, M.E., Villari, F., Costerton, J.W., 1991. Determination of the coefficient of kinetic friction of urinary catheter materials. J. Urol. 145, 610-612.

Lee, S., Spencer, N.D., 2005. Aqueous lubrication of polymers: Influence of surface modification. Tribol. Int. 38, 922-930.

Makamba, H., Kim, J.H., Lim, K., Park, N., Hahn, J.H., 2003. Surface modification of poly(dimethylsiloxane) microchannels. Electrophoresis. 24, 3607-3619.

Mark, J.E., 2009. The Polymer Data Handbook second ed. Oxford University Press, New York. 


\section{ACCEPTED MANUSCRIPT}

Madsen, N.J., 2010. Method for sterilising a medical device having a hydrophilic coating. U.S. Patent, US 7,833,475 B2.

Marmieri, G., Pettenati, M., Cassinelli, C., Morat, M., 1996. Evaluation of slipperiness of catheter surfaces. J. Biomed. Mater. Res. A 33, 29-33.

Nickel, J.C., Olson, M.E., Costerton, J.W., 1987. In vitro coefficient of kinetic friction: study of urinary catheter biocompatibility, Urology 24, 501-503.

Palchesko, R.N., Zhang, L., Sun, Y., Feinberg, A.W., 2012. Development of polydimethylsiloxane substrates with tunable elastic modulus to study cell mechanobiology in muscle and nerve. PLOS One 7, e51499.

Røn, T., Chronakis, I.S., Lee, S., 2014. Lubrication of soft and hard interfaces with thermoresponsive F127 hydrogel. Polymer 55, 5708-5717.

Røn, T., Lee, S., 2016. In vitro urethra model to characterize the frictional properties of urinary catheters, Proceedings for $12^{\text {th }}$ IASTED International Conference Biomedical Engineering, 188194.

Stensballe, J., Looms, D., Nielsen, P.N., Tvede, M., 2005. Hydrophilic-coated catheters for intermittent catheterisation reduce urethral micro trauma: a prospective, randomised, participantblinded, crossover study of three different types of catheters. Eur. Urol. 48, 978-983.

Tunney, M.M., Gorman, S.P., 2002. Evaluation of poly(vinyl pyrollidone)-coated biomaterials for urological use. Biomaterials 23, 4601-4608.

Uyama, Y., Tadokoro, H., Ikada, Y., 1990. Surface lubrication of polymer films by photoinduced graft polymerization. J. Appl. Polym. Sci. 39, 489-498.

Uyama, Y., Tadokoro, H., Ikada, Y., 1991. Low-frictional catheter materials by photo-induced graft polymerization. Biomaterials 12, 71-75. 
Ikada, Y., Uyama, Y., 1993. Lubricating Polymeric Surfaces. Technomic Publishing Co., Inc. Lancaster, Basel.

G. Wanka, H. Hoffmann, and W. Ulbricht, 1994. Phase diagrams and aggregation behavior of poly(oxyethylene)-poly(oxypropylene)-poly(oxyethylene) triblock copolymers in aqueous solutions. Macromolecules 27, 4145-4159.

Wilson, M., 2013. Catheter lubrication and fixation: interventions, British J. Nursing 22, 566-569.

Young, W.C., Budynas, R.G., Sadegh, A.M., 2012. Roark's Formulas for Stress and Strain, seventh ed. McGraw-Hill, New York.

Zhou, J., Khodakov, D.A., Ellis, A.V., Voelcker, N.H., 2012. Surface modification for PDMSbased microfluidic devices. Electrophoresis 33, 89-104.

Report

on

CuriCath ${ }^{\circledR}$

http://www.curion.nl/zdjecia/file/PDF\%20folders/DSM\%20rapport\%20CuriCat.pdf

SpeediCath ${ }^{\circledR}$

Product

Monograph

http://www.coloplast.ca/Global/3_Bladder\%20and\%20Bowel/Pdf/New\%20Coloplast\%20monograp h.pdf 


\section{Highlights}

- A new friction tester for urinary catheters was developed

- Based on balance force sensor, both pushing and friction forces were detected

- Silicone-based urethra was superior to agar gel in distinction of different catheters

- The new tester evaluates both surface coatings and tubing materials of catheters 